Article

\title{
Size-Controllable Synthesis of Zeolitic Imidazolate Framework/Carbon Nanotube Composites
}

\author{
Fang Fu ${ }^{1}$, Bin Zheng ${ }^{1, * \mathbb{C}}$, Lin-Hua Xie ${ }^{2}{ }^{\mathbb{D}}$, Huiling Du ${ }^{1}$, Shuangming $\mathrm{Du}^{1}$ and Zhenhua Dong ${ }^{3}$ \\ 1 School of Materials Science and Engineering, Xi'an University of Science and Technology, Xi'an 710054, \\ China; nicolehuizheng@gmail.com (F.F.); hldu@xust.edu.cn (H.D.); smdu@xust.edu.cn (S.D.) \\ 2 Beijing Key Laboratory for Green Catalysis and Separation, College of Environmental and Energy \\ Engineering, Beijing University of Technology, Beijing 100124, China; xielinhua@bjut.edu.cn \\ 3 College of Chemistry, Chemical and Environmental Engineering, Henan University of Technology, \\ Zhenzhou 450001, China; zhdong@haut.edu.cn \\ * Correspondence: zhengbin@xust.edu.cn; Tel.: +86-131-8601-0398
}

Received: 26 August 2018; Accepted: 19 September 2018; Published: 20 September 2018

check for updates

\begin{abstract}
Composite materials that combine the unique properties of zeolitic imidazolate frameworks (ZIFs) and carbon nanotubes (CNTs) can give rise to novel applications. Here, ZIF-8/CNT composites were successfully prepared with and without the addition of an agent template. The size of the ZIF-8 crystals in the composite materials was controlled by varying the template, feeding order, and concentration of reactants. Thus, ZIF- 8 crystals with a wide variety of sizes (from nano- to micrometer size, which is range that differs by a factor of 10) were obtained, depending on the conditions. This size-controllable synthesis of ZIF-8 was achieved by modifying the number of nucleation sites on the CNTs, as revealed by density functional theory (DFT) calculations. This work provides an efficient method for preparing ZIF-8/CNT composites with controllable size and can pave the way for the synthesis of other metal-organic framework (MOF)/CNT composite materials.
\end{abstract}

Keywords: crystal growth; ZIF-8; carbon nanotube; DFT calculation

\section{Introduction}

Zeolitic imidazolate frameworks (ZIFs), which is a subfamily of metal-organic frameworks (MOFs), have attracted great attention because of their tunable pore metrics and chemical functionality [1-3]. However, the tendency of ZIFs to form isolated particles and the poor conductivity of ZIF crystals limit the applications of these materials in a variety of applications, including mixture separation and battery electrodes.. In this sense, carbon nanotubes (CNTs), which have remarkable electrical, thermal, optical, and mechanical properties [4-6], might be a good candidate to be incorporated into ZIFs, enabling the integration of the unique properties of both types of materials. Therefore, increasing research efforts have been made to develop ZIF/CNT composites.

Among all known ZIF materials, ZIF-8 has been widely studied because its low cost, easy preparation, and exceptional hydrothermal stability [7] make it compatible with polymers [8-10], graphene [11], graphene oxide (GO) [12-17], graphene quantum dots (GQDs) [18], carbon nanotubes (CNTs) [19-23], etc. Enormous efforts have been made to synthesize ZIF-8/CNT composites. The presence of anchoring groups such as hydroxyl, epoxy, or carboxyl groups on the template material is critical for the formation of ZIF-8 hybrid composites. The CNTs were treated using an acid mixture to graft oxidized carboxylic groups which can anchor the organic linkers of MOFs [24,25]. Also, the CNTs were oxidized to form a negatively charged surface, which can adsorb positively charged metal ions of MOFs [26]. A continuous, inter-grown ZIF-8 membrane on the surface of CNTs was obtained via the secondary growth method [19]. Zinc precursors ( $\mathrm{ZnO}$ nanoparticles) were put on 
the surface of CNTs and in-situ growth of ZIF-8 in the presence of CNTs were achieved [27]. Because of compatibility, polymers (such as PVP and Polydopamine) were used to wrap the CNTs before the growth of ZIF-8 [21,28,29]. A ZIF-8/CNT "necklace" composite or core-shell structures were obtained.

As a result of this functionalization, CNTs can undergo important changes and lose their original properties, for example, they can become hydrophilic. This is undesirable for some applications that require CNTs with specific properties. Thus, the preparation of ZIF-8/unfunctionalized-CNT composites is necessary. In addition, efficient applications require these materials to be prepared in a controllable fashion. For instance, small ZIF-8 particles are preferable for Li-S batteries because of their higher sulfur utilization [30]. In the case of mixture separation applications, large ZIF-8 crystals can reduce the number of undesirable inter-grain gaps. Thus, size-controllable synthesis of ZIF/CNT composites is valuable. Control of ZIF-8 crystal size has been achieved while using a mixed-metal approach, and the mean size of nanoparticles increased monotonically with an increase in cobalt(II) dopant concentration [31]. Also, adding 1-methylimidazole at different concentration can lead to the formation of size-tunable ZIF-8 particles [32]. In this work, unfunctionalized multi-walled (MW) CNTs were successfully inserted into ZIF-8 crystals to form ZIF-8/CNT composite materials. In the preparation method, non-ionic, anionic, or cationic agents were used as a third-party template for growing ZIF-8 on the CNTs. The feeding order of $\mathrm{Zn}^{2+}$ and 2-methyl imidazolate solutions was investigated with the aim of controlling the size of the ZIF-8 crystals.

\section{Materials and Methods}

\subsection{Materials}

MWCNTs (>95\%; inner diameter: 3-5 nm; outer diameter: $8-15 \mathrm{~nm}$; length: ca. $50 \mu \mathrm{m}$; Aladdin) and methanol ( $>99.5 \%$; Aladdin) were used as received. PVP (Mw: ca. 8000$)$, sodium dodecyl sulfate (SDS) (99\%; Mw: ca. 288), cetyltrimethylammonium bromide (CTAB; 99\%; Mw: ca. 364), 2-methylimidazole (2-MeIm; 98\%), and zinc nitrate hexahydrate (99\%) were purchased from Aladdin (Shanghai, China).

\subsection{Synthesis of ZIF-8 and ZIF-8/CNT Composites}

ZIF-8 was prepared while using a procedure that is detailed in our previous work [33]. A solution of $\mathrm{Zn}\left(\mathrm{NO}_{3}\right)_{2} \cdot 6 \mathrm{H}_{2} \mathrm{O}(0.4507 \mathrm{~g}, 1.5 \mathrm{mmol})$ in $20 \mathrm{~mL}$ of methanol was added to $20 \mathrm{~mL}$ of 2-MeIm $(0.2513 \mathrm{~g}$, $3.0 \mathrm{mmol}$ ) methanol solution. The mixture was stirred for $5 \mathrm{~min}$ at $1000 \mathrm{rpm}$ and left undisturbed at room temperature for $24 \mathrm{~h}$. The as-formed ZIF- 8 crystals were collected via centrifugation, followed by repeated washing with methanol, and the final product was vacuum-dried at $60^{\circ} \mathrm{C}$.

ZIF-8/CNT composites were fabricated using the following in situ ZIF-8 synthesis (Scheme 1). The agents $(0.1 \mathrm{~g}$ of PVP, SDS, or CTAB) were dissolved in methanol $(20 \mathrm{~mL})$ with stirring. CNTs (15 mg) were subsequently added to the above solution, sonicated in a bath-type sonicator (KQ2200) for $0.5 \mathrm{~h}$, and stirred for $2 \mathrm{~h}$. As a conventional feeding order [1,3], 2-MeIm ( $3.0 \mathrm{mmol})$ was added to the as-prepared CNT solution with stirring for $0.5 \mathrm{~h}$, and $20 \mathrm{~mL}$ of a methanol solution of $\mathrm{Zn}\left(\mathrm{NO}_{3}\right)_{2} \cdot 6 \mathrm{H}_{2} \mathrm{O}$ $(1.5 \mathrm{mmol})$ was added to the dispersion. The mixture was stirred for $5 \mathrm{~min}$ at $1000 \mathrm{rpm}$ and left undisturbed at room temperature for $24 \mathrm{~h}$. The generated precipitates were washed several times with methanol, collected after centrifugation, and vacuum dried at $60^{\circ} \mathrm{C}$. 


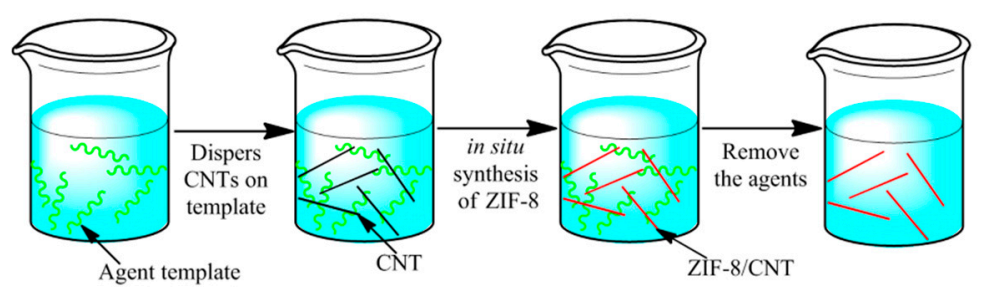

Scheme 1. Schematic drawing of the synthesis approach used to prepare zeolitic imidazolate framework-8/carbon nanotubes (ZIF-8/CNT) composite materials.

Table 1 shows the summary for the synthesis of ZIF-8/CNT composites in this work. When compared to the reported works $[19,20,22]$, the CNTs used in this study were unmodified. The synthesis was achieved by feeding the CNTs into the agent solution, which then acts as the template and did not wrap the CNTs. CNTs wrapped with polymers were usually obtained by pouring polymer into the CNT solution. The polymer molecules were more mobile because of their concentration gradient in CNT solution. Thus, there are more chances for polymer molecules to meet and wrap around the CNTs. For comparison, the polymer solution in our study was left undisturbed at room temperature. Before adding the CNT solution, an equilibrium network between neighboring polymer molecules was formed via nonbonded interactions (VDW and electrostatic). The polymer network serves as the function of supporting free CNTs instead of wrapping them. Another point in the current experimental design (Table 1) is the feed order of $\mathrm{Zn}^{2+}$ and 2-methyl imidazolate solutions to alter the size and shape of the ZIF-8 crystals. This approach is extremely simple and convenient.

Table 1. Summary table for synthesis of ZIF-8/CNT composites. Reaction temperature and time are $298 \mathrm{~K}$ and $24 \mathrm{~h}$, respectively. Values inside parentheses are the optional amounts of 2-MeIm.

\begin{tabular}{ccccc}
\hline Feeding Order 1 & Agent & CNT (mg) & Zn(NO $)_{\mathbf{2}}$ (mmol) & 2-MeIm (mmol) \\
\hline & - & 15 & 1.5 & 3.0 \\
& PVP & 15 & 1.5 & 3.0 \\
& SDS & 15 & 1.5 & 3.0 \\
& CTAB & 15 & 1.5 & 3.0 \\
\hline Feeding Order 2 & Agent & CNT (mg) & 2-MeIm (mmol) & Zn( $\left.\mathbf{N O}_{3}\right)_{\mathbf{2}}$ (mmol) \\
\hline & - & 15 & 3.0 & 1.5 \\
& PVP & 15 & $3.0(105)$ & 1.5 \\
& SDS & 15 & $3.0(105)$ & 1.5 \\
\hline
\end{tabular}

\subsection{Measurements}

Powder X-ray diffraction (PXRD) patterns of ZIF-8 and the composites were obtained on a Bruker Advanced X-ray diffractometer (Shimadzu, Kyoto, Japan) $(40 \mathrm{kV}, 30 \mathrm{~mA})$ with $\mathrm{Cu} \mathrm{K} \alpha(\lambda=0.15406 \mathrm{~nm})$ radiation at a scanning rate of $2^{\circ} \mathrm{min}^{-1}$ from 5 to $40^{\circ}$. Morphologies of the synthesized ZIF- 8 and ZIF-8/CNT composite materials were characterized while using scanning electron microscopy (SEM) (Carl Zeiss AG, Jena, Germany) on a S-4800 microscope (Hitachi) with an accelerating voltage of $5.0 \mathrm{kV}$. ZIF-8 pellets were prepared for Fourier transform infrared (FTIR) spectroscopy measurements, which were taken on a Spectrum GX device (Perkin-Elmer, Waltham, USA). Sorption isotherms for nitrogen were measured with an automatic volumetric adsorption apparatus (Micrometrics ASAP 2020, Shanghai, China) at $77 \mathrm{~K}$. The as-synthesized samples (weight $37-45 \mathrm{mg}$ ) were placed in a quartz tube and then activated under high vacuum at $403 \mathrm{~K}$ for $5 \mathrm{~h}$ to remove methanol and other volatile compounds before measurements were made. 


\subsection{Theoretical Calculations}

Single layer graphene was used as a computational model for a small area of CNT. We used a supercell that consisted of a single layer of graphene with $4 \times 4$ primitive cells and a $30 \AA$ vacuum space perpendicular to the graphene. Three lattice sites were suggested as possible binding positions upon the adsorption of $\mathrm{Zn}$ atoms or 2-MeIM groups on graphene (Figure S3). Perdew-Burke-Ernzerhof parameterization of the generalized gradient approximation [34] and projector augmented wave (PAW) $[35,36]$ potentials, as implemented in the Vienna ab initio simulation package (VASP) [37-40] were used. Gamma point sampling was used with a cut off energy of $600 \mathrm{eV}$. The binding energy of guests on a graphene sheet was defined as:

$$
\Delta E=\left(E_{\mathrm{C}}+E_{\mathrm{Zn} \text { or 2-MeIM }}\right)-E_{\mathrm{Zn} / \mathrm{C} \text { or 2-MeIM/C, }}
$$

where $\Delta E$ is the binding energy, $E_{\mathrm{C}}$ is the energy of the single layer of graphene, and $E_{\mathrm{Zn}}$ and $E_{2-\mathrm{MeIM}}$ are the energies of a single zinc atom and a single 2-MeIm group, respectively, and $E_{\mathrm{Zn} / \mathrm{C}}$ or $E_{2-\mathrm{MeIM} / \mathrm{C}}$ correspond to the energies of the composite system.

\section{Results}

Figure 1 shows PXRD patterns of ZIF-8 and the ZIF-8/CNT composite. The diffraction pattern of the ZIF-8/CNT composite was similar to that of pure ZIF-8, revealing that the crystal structure of ZIF-8 was preserved upon the incorporation of CNT following our synthesis approach. The PXRD peak of CNTs $\left(2 \theta=26^{\circ}\right)$ was not observed (Figure 1), and this can be ascribed to the lower intensity of this signal as compared to the diffraction signal of ZIF-8 $[19,28,29]$.

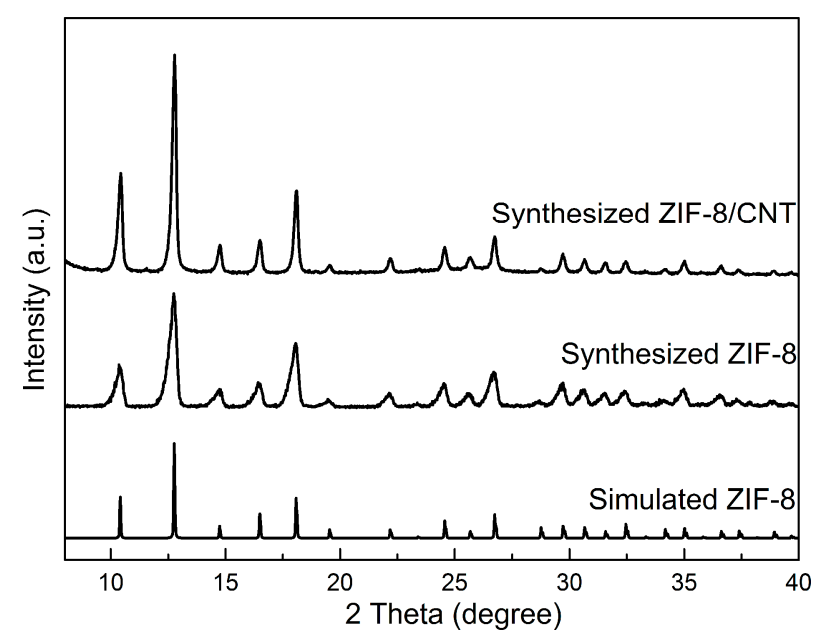

Figure 1. Powder X-ray diffraction (PXRD) patterns of the simulated ZIF-8, synthesized ZIF-8, and ZIF-8/CNT composite.

PXRD patterns for all of samples are shown in Figure S1, which shows that the relative crystallinity of ZIF-8 is extremely strong. Still, there are small peaks for the side product $\left(\mathrm{Zn}_{3}(\mathrm{OH})_{4}\left(\mathrm{NO}_{3}\right)_{2}\right)$ and reactant $\left(\mathrm{Zn}\left(\mathrm{NO}_{3}\right)_{2} \cdot 6 \mathrm{H}_{2} \mathrm{O}\right)$ in some samples (Figure $\left.\mathrm{S} 2\right)$. It is believed that the small amount of the minor phases here has negligible effects on the structure and properties of the ZIF-8/CNT composites. $\mathrm{N}_{2}$ sorption experiments show that the micropore volume of ZIF-8/CNT is $0.42-0.46 \mathrm{~cm}^{3} \cdot \mathrm{g}^{-1}$, which is larger than the reported micropore volume $\left(\sim 0.32 \mathrm{~cm}^{3} \cdot \mathrm{g}^{-1}\right)[41,42]$ in the presence of some by-products or amorphous compounds. Furthermore, the hydrothermal synthesis of ZIF-8 seems to be easily generated by-products, where the minimum Hmim/Zn molar ratio is 20 [43]. Using a smaller ratio $(\mathrm{Hmim} / \mathrm{Zn}=8)$ in the solvothermal (methanol) synthesis of ZIF-8 leads to the preparation of pure ZIF-8 nanocrystals [44]. The Hmim/Zn molar ratio was even reduced to 2 (methanol) [3] and 0.9 (DMF) [1], 
and large, but still pure microcrystals were produced. All of these reported experimental results support our choice of using a low Hmim/Zn molar ratio (2) in the solvothermal method.

Morphologies of ZIF-8 and the ZIF-8/CNT composites are shown in Figure 2. In pure ZIF-8, large rhombic dodecahedral particles were observed (Figure 1a). Upon the incorporation of CNTs, the ZIF-8 crystals were reduced in size to the nanoscale level (Figure $2 b$ ). CNTs were found to be inserted ZIF- 8 single crystals from multiple directions (Figure 2c), while preserving the crystal structure of ZIF-8.
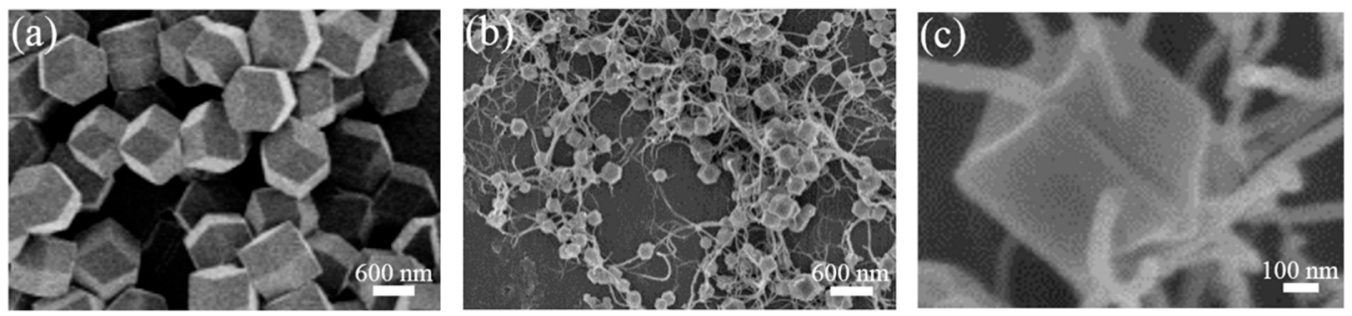

Figure 2. SEM images of: (a) ZIF-8 and (b) ZIF-8/CNT. (c) Enlarged SEM image of ZIF-8/CNT.

Different additive agents were used to synthesize the ZIF-8/CNT composite materials. Small-sized ZIF-8 crystals (ca. $100 \mathrm{~nm}$ ) were obtained by adding a non-ionic agent (PVP), resulting in ZIF-8/CNT composites that had a necklace-like morphology (Figure 3a). In comparison, the addition of anionic (SDS) or cationic (CTAB) agents resulted in larger sized ZIF-8 crystals (Figure 3 and Figure S4).

The additive agents play two roles during the synthesis of the ZIF-8/CNT composite materials. On one hand, the additive agents act as sites for nucleation and growth of ZIF- 8 crystals, as reported in the synthesis of nanowires [45] and metal nanoparticles@ZIF-8 core-shell structures [46]. Lu et al. [46] revealed the presence of coordination (or hydrophobic) interactions between the terminal groups of polymers and metal atoms (or organic linkers) of MOFs. For example, in PVP, the pyrrolidone rings $(\mathrm{C}=\mathrm{O})$ can coordinate with zinc atoms in ZIF nodes, and apolar groups of PVP can adsorb 2-MeIm linkers via hydrophobic interactions. On the other hand, additive agents can be good dispersants for CNTs [47]. Here, the additive agents do not have a direct effect on the incorporation of low amounts of CNTs in the final product, as revealed by FTIR spectroscopy (Figure S5). Well-dispersed CNTs that result with the use of PVP can increase the number of nucleation sites for ZIF- 8 crystals, which decreases the size of the resultant ZIF-8 crystals (Figure 3a). When compared to acid- [19] and PVP-functionalized CNTs [20], bare CNTs generated less nucleation sites, which resulted in larger ZIF-8 crystals.
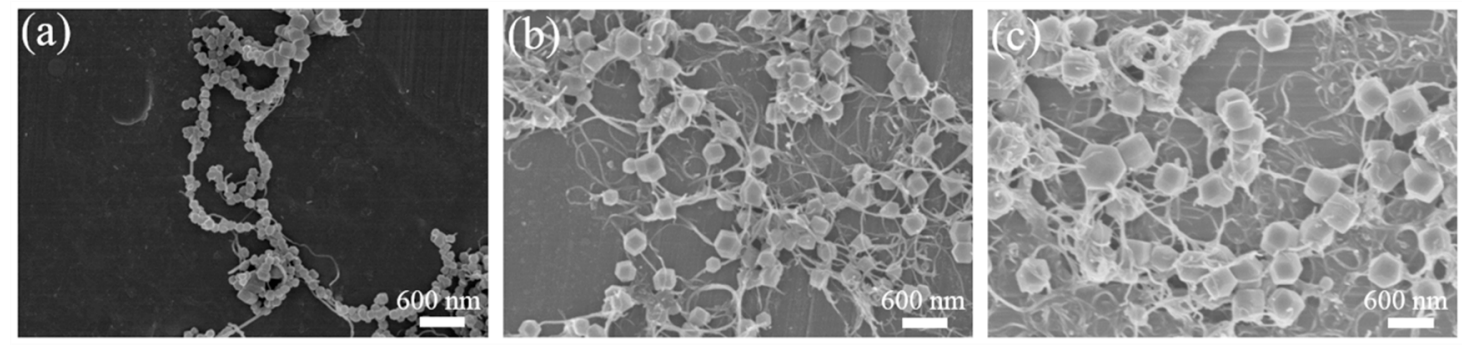

Figure 3. SEM images of the ZIF-8/CNT composite materials prepared by adding: (a) PVP, (b) sodium dodecyl sulfate (SDS), and (c) cetyltrimethylammonium bromide (CTAB).

In-situ synthesis of ZIF-8/CNT composite materials was conducted. The feed order of the 2-MeIm and $\mathrm{Zn}\left(\mathrm{NO}_{3}\right)_{2} \cdot 6 \mathrm{H}_{2} \mathrm{O}$ solutions was considered here. Adding 2-MeIm into the mixed solution of $\mathrm{Zn}\left(\mathrm{NO}_{3}\right)_{2} \cdot 6 \mathrm{H}_{2} \mathrm{O}$ and CNTs resulted in larger ZIF- 8 crystals (Figure 4). In particular, PVP agents can be used to prepare ZIF-8 crystals with a wide range of sizes. Li et al. [22] also synthesized ZIF-8/CNT composites with large-sized ZIF-8 crystals ( $500 \mathrm{~nm}$ ). In comparison, we obtained micrometer-sized ZIF-8 crystals when using bare CNTs in this work (Figure $4 b$ ). 

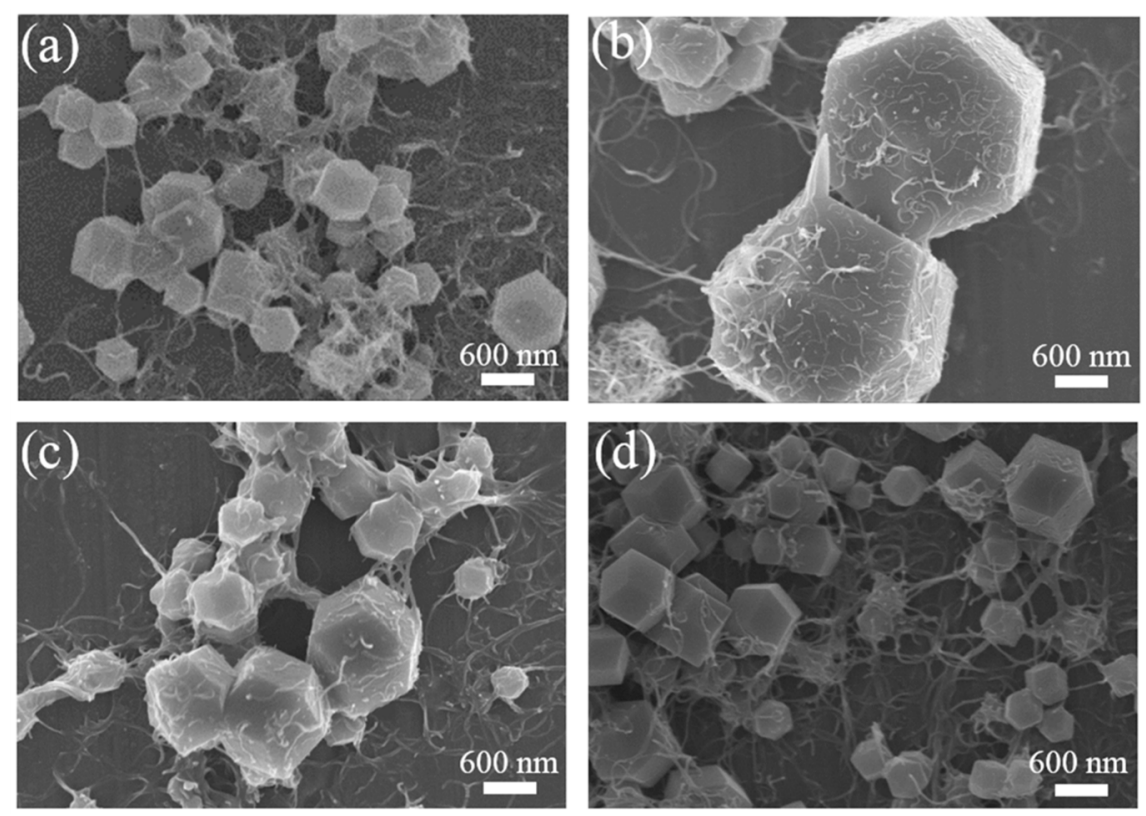

Figure 4. SEM images of the ZIF-8/CNT composite materials prepared by mixing CNTs with $\mathrm{Zn}\left(\mathrm{NO}_{3}\right)_{2} \cdot 6 \mathrm{H}_{2} \mathrm{O}$ solution followed by the addition of a 2-MeIm solution: (a) no additive agents added, (b) PVP, (c) SDS, and (d) CTAB added.

We found that the feed order of the reactants plays an important role in controlling the size of ZIF-8 crystals. In fact, the feed order can even change the topology of the synthesized MOFs. For example, adding the metal ion solution into the ligand solution that was premixed with a hydrophobic template led to the synthesis of the metal azolate framework (MAF)-6 with RHO topology, and with a different feed order, MAF-5 with ANA topology can be obtained [48]. The difference in the template effect (hydrophobicity) that results because of feed order were used to understand the different topologies of the synthesized hydrophobic MOFs. In this work, instead of chemical function, the templates serve a function of dispersing bare CNTs where ZIF- 8 crystals nucleate and grow to form composite materials.

Larger ZIF-8 crystals in composite materials reveal a lower number of nucleation sites. With mixing the $\mathrm{CNTs}$ and $\mathrm{Zn}\left(\mathrm{NO}_{3}\right)_{2} \cdot 6 \mathrm{H}_{2} \mathrm{O}$ solution, $\mathrm{Zn}^{2+}$ is expected to bond the surface carbon atoms of CNTs, which serves as nucleation and growth centers for ZIF-8 crystals. We computed the binding energy between $\mathrm{Zn}$ and CNT (Figure 5a). Among the three possible adsorption sites (i.e., top, center, and middle positions), only the center position (distance: $3.58 \AA$ and binding energy: $5.7 \mathrm{Kcal} \mathrm{mol}^{-1}$ ) showed strong adsorption. Thus, when adsorbed on the top and middle positions, $\mathrm{Zn}$ showed weak physical adsorption energies. Because these species are easily desorbed, they would not likely serve as nucleation centers. In comparison, the 2-MeIm group is strongly bound to the surface of the CNT for the three possible adsorption positions (2.88-3.18 $\AA$ and $6.3-7.4 \mathrm{Kcal} / \mathrm{mol})$. Therefore, more nucleation sites are generated when using 2-MeIm-functionalized CNTs. 


\section{Top position Center position Middle position}

(a)

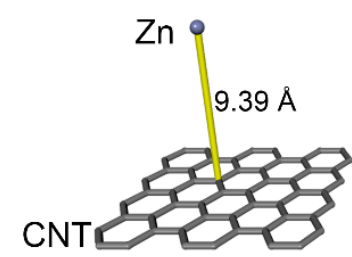

Binding Energy $=0.9 \mathrm{Kcal} / \mathrm{mol}$

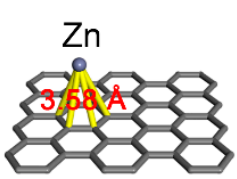

$5.7 \mathrm{Kcal} / \mathrm{mol}$

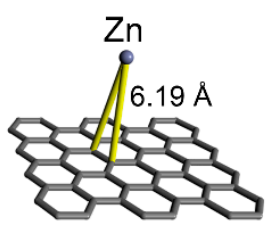

$1.1 \mathrm{Kcal} / \mathrm{mol}$

(b)
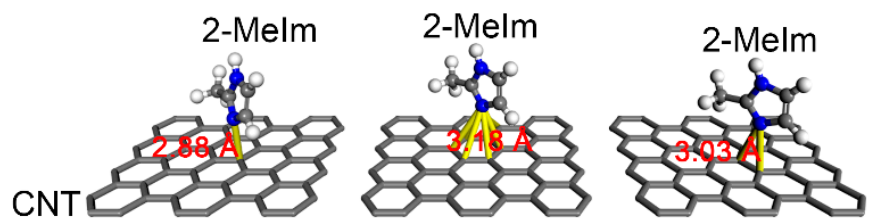

Binding Energy $=6.6 \mathrm{Kcal} / \mathrm{mol}$

$6.3 \mathrm{Kcal} / \mathrm{mol}$

Figure 5. Computed binding energies for (a) $\mathrm{Zn}$ and (b) 2-MeIm on the surface of CNTs.

According to the above discussion, the ZIF-8 crystal size in ZIF-8/CNT composites can be tuned by reducing/increasing the number of nucleation sites on the CNTs. We further confirmed this hypothesis by adjusting the ratio of 2-MeIm to $\mathrm{Zn}^{2+}$. According to the chemical formula of ZIF-8, the ideal 2-MeIm $/ \mathrm{Zn}^{2+}$ ratio is 2. Higher 2-MeIm $/ \mathrm{Zn}^{2+}$ ratios result in a larger number of adsorbed 2-MeIm groups on the CNTs, and these can subsequently serve as nucleation sites for ZIF-8 crystals. Thus, composite materials with small ZIF- 8 crystal size were obtained upon increasing the ratio of 2-MeIm to $\mathrm{Zn}^{2+}$ to 70 (Figure 6). A wide variety of ZIF-8 single crystal sizes were obtained herein, with the largest crystals $\left(\mathrm{PVP}, 2-\mathrm{MeIm}: \mathrm{Zn}^{2+}=2: 1\right)$ being at least 10 times greater than those that were obtained with SDS or CTAB and a 2-MeIm $/ \mathrm{Zn}^{2+}$ ratio of 70 . Also, the average size of ZIF- 8 crystals prepared using a 2-MeIm $/ \mathrm{Zn}^{2+}$ of 70 without any agent is about $104 \mathrm{~nm}$ (Figure S7). This size vale is the same as that of the cases with agents $(108-110 \mathrm{~nm}$ in the low row of Figure 6). This indicates the effect of an additive agent on the size of ZIF- 8 crystals that were prepared using a high molar ratio of 2-MeIm to $\mathrm{Zn}$ is negligible.

When the 2-MeIm $/ \mathrm{Zn}^{2+}$ ratio was set at 2, the PVP-assisted ZIF-8/CNT composite has a smaller size when compared to SDS- and CTAB- assisted ZIF-8/CNT composites (middle panels of Figure 6). This can be understood by the preference for ZIF- 8 growing on the surface of PVP molecules. It has been reported that there are strong interactions (coordination and hydrophobic) between PVP and reactants ( $\mathrm{Zn}$ and 2-MeIm) [48]. The consumption of reactants by PVP may limit the size of ZIF crystals that are attached to CNTs. 

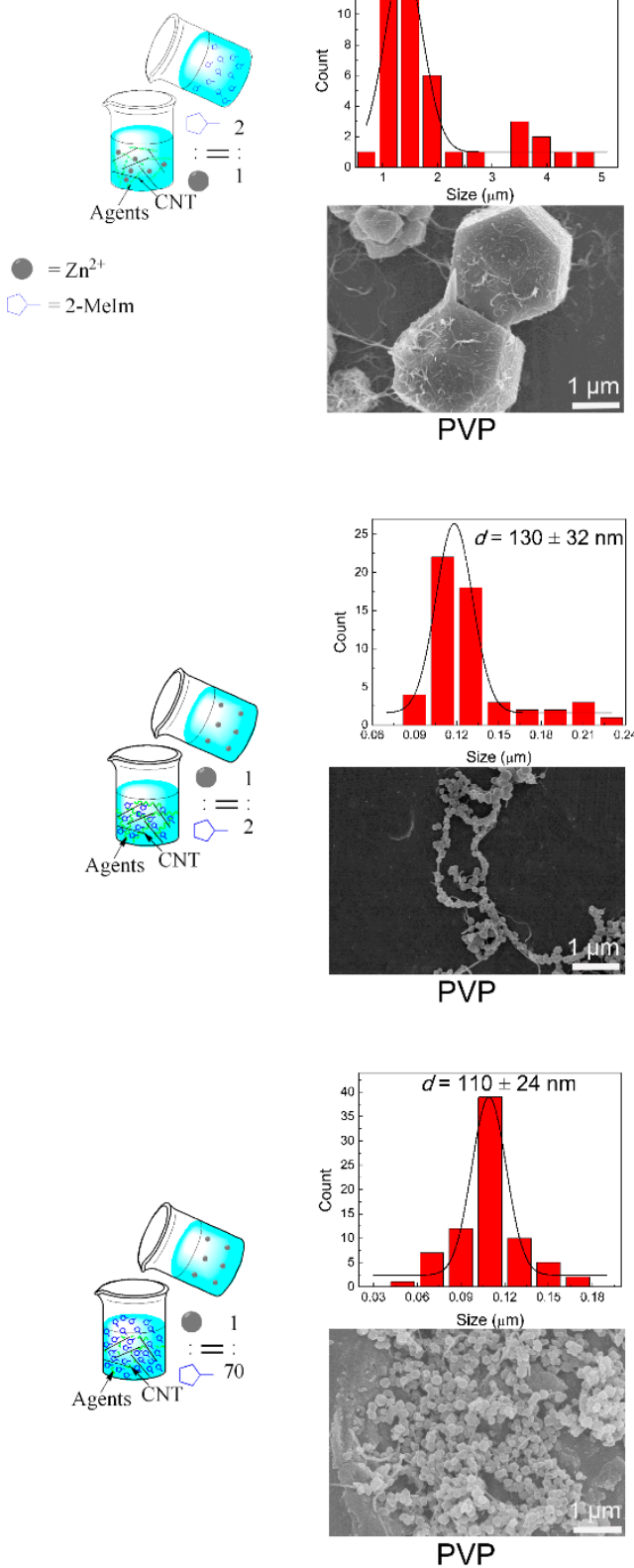

PVP
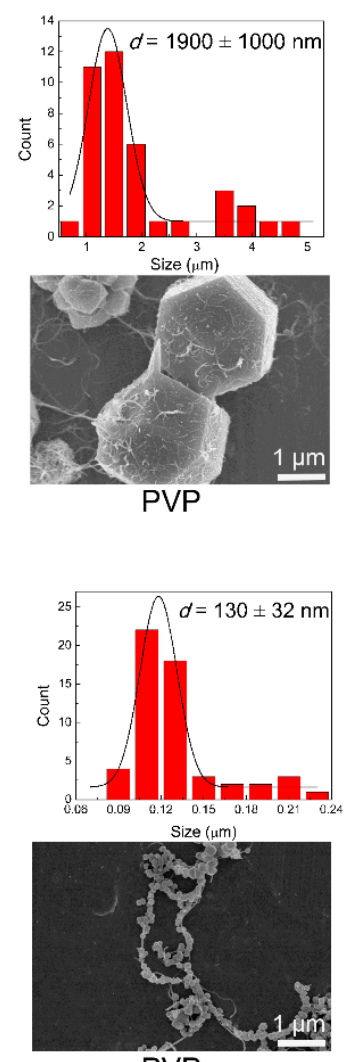

PVP
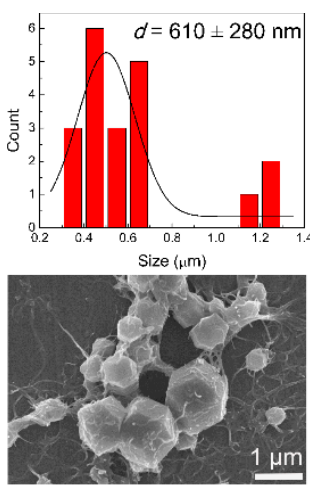

SDS
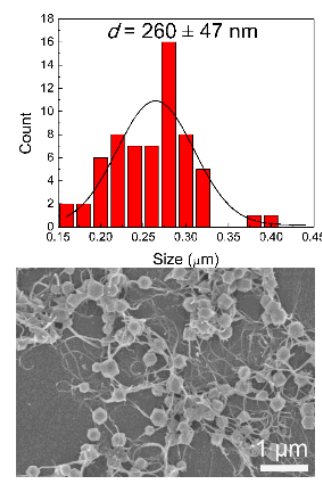

SDS

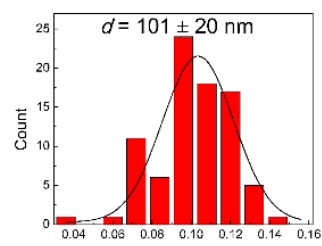

Size $(\mu \mathrm{m})$

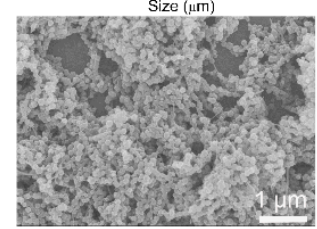

SDS
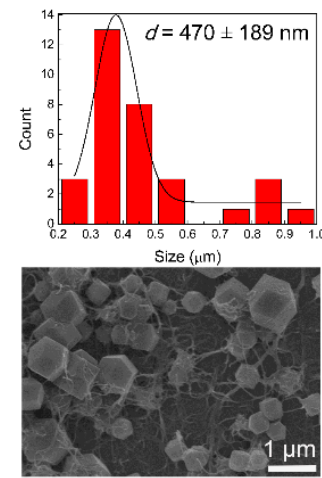

CTAB
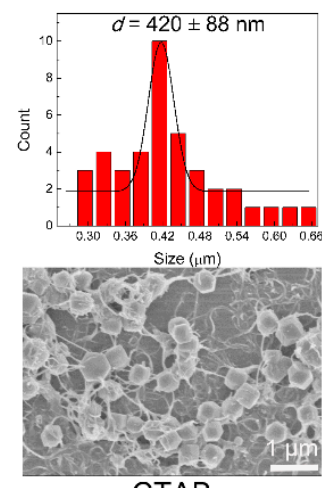

CTAB
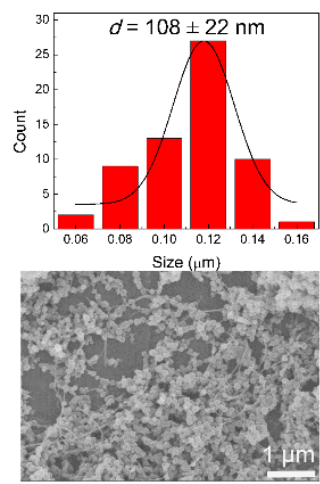

CTAB

Figure 6. SEM images with size distribution histograms for ZIF-8/CNT composite materials prepared under different synthesis conditions. The size distributions histogram for large ZIF-8 crystals (top-left corner) was extracted from Figure S6 (large observation area).

Nitrogen sorption experiments at $77 \mathrm{~K}$ were conducted to check the porosity of ZIF-8/CNT composite materials. Isotherms for one typical series of composites that has clearly distinct ZIF- 8 crystal size (upper panels in Figure 6) are presented in Figure 7. The shapes of the isotherms (Figure 7) for ZIF-8/CNT composites have a significant hysteresis in the adsorption-desorption process, which is indicative of a type-IV isotherm. Pure ZIF-8 and pure CNT show type-IV and type-II adsorption isotherms, respectively. In Figure 7, the uptake of nitrogen at a relative pressure of 0.1 is located in the range of $270-300 \mathrm{~cm}^{3} \cdot \mathrm{g}^{-1}$, which is due to micropore filling from the enhanced adsorbent-adsorbate interactions in the micropores. For comparison, the uptake amounts at low pressures by pure ZIF-8 and pure MWCNT were $400-500$ and $60-70 \mathrm{~cm}^{3} \cdot \mathrm{g}^{-1}$, respectively (Table 2). The above observations indicate that the adsorption properties of the ZIF-8/CNT composite are closer to ZIF-8. 
From Table 2, the highest Brunauer-Emmett-Teller (BET) surface area and microporous volume in ZIF-8/CNT composite correspond to medium-sized ZIF-8 crystal (SDS in the upper panels of Figure 6). This indicates that the size dependence of the internal surface area and microporous volume is not obvious, and this was also observed with different sizes of Co/ZIF-8 nanoparticles [31]. Furthermore, a similar external surface area $\left(\sim 26.0 \mathrm{~m}^{2} \cdot \mathrm{g}^{-1}\right)$ was obtained for the three samples in Figure 7. However, in pure ZIF-8 and its derivatives, the external surface area decreased with an increase in crystal size [31]. The unusual observation here can be understood in terms of the incorporation of CNT, which made a significant contribution to the external surface area in composites. In the large ZIF-8 crystals, CNT partly inserts into crystals and leaves tentacles on the surface (Figure 6). Importantly, large ZIF-8 crystals do not allow the insertion of many CNTs because of potential structure instability from the induction of defects. This can result in a large exposed surface area of CNT. However, small ZIF-8 crystals prefer to attach to the surface of CNT, and then core/shell morphology can be formed (Figure 6). The wrapped CNT core contributes less to the total external surface area of composites. Thus, the exposed surface area of CNT in composites increases with an increase in ZIF-8 crystal size. This increase compensates for the loss of external surface area, which results from large sized nanoparticles.

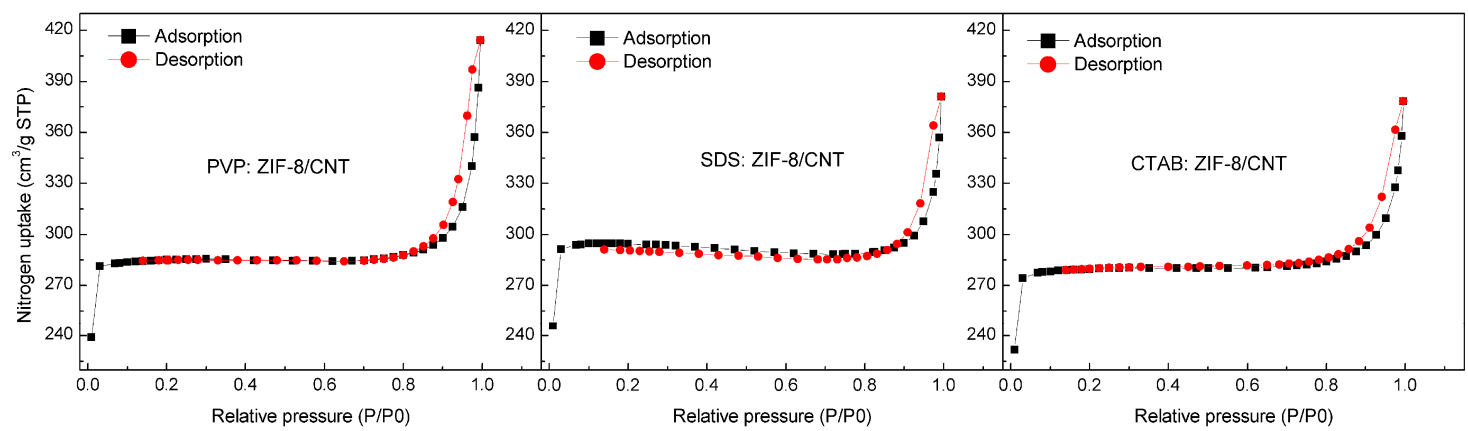

Figure 7. Nitrogen adsorption-desorption isotherms at $77 \mathrm{~K}$ for ZIF-8/CNT composites prepared by adding different agents (PVP, SDS, and CTAB).

Table 2. Comparison of values for BET surface $\left(S_{\mathrm{BET}}\right)$, microporous volume $\left(V_{\text {micro }}\right), \mathrm{N}_{2}$ uptake $(\mathrm{p} / \mathrm{p} 0=0.1)$ for ZIF-8, ZIF-8/CNT composites prepared with different adding agents (PVP, SDS, and $(\mathrm{TAB})$, and pure CNT.

\begin{tabular}{cccc}
\hline Sample & $S_{\text {BET }}\left(\mathbf{m}^{\mathbf{2}} \mathbf{g}^{-\mathbf{1}}\right)$ & $V_{\text {micro }}\left(\mathbf{c m}^{\mathbf{3}} \cdot \mathbf{g}^{-\mathbf{1}}\right)$ & $\mathbf{N}_{\mathbf{2}}$ Uptake $(\mathbf{P} / \mathbf{P 0}=\mathbf{0 . 1})\left(\mathbf{c m}^{\mathbf{3}} \cdot \mathbf{g}^{-\mathbf{1}}\right)$ \\
\hline Pure ZIF-8 [31] & 1410 & 0.546 & $400-500$ \\
PVP: ZIF-8/CNT & 945 & 0.429 & 280 \\
SDS: ZIF-8/CNT & 976 & 0.459 & 294 \\
CTAB: ZIF-8/CNT & 928 & 0.421 & 278 \\
Pure MWCNT [49] & 240 & - & $60-70$ \\
\hline
\end{tabular}

\section{Conclusions}

In conclusion, ZIF-8/CNT composites were successfully prepared via in situ synthesis of ZIF-8 on bare CNTs. By controlling the feed order of the $\mathrm{Zn}^{2+}$ and 2-MeIm solutions, we were able to tune the size of the ZIF-8 crystals in the composites from the nano-meter scale to micro-meter scale. This was explained by the weak binding between $\mathrm{Zn}$ and CNTs, when compared to that between 2-MeIm and $\mathrm{CNTs}$. Mixing CNTs and the $\mathrm{Zn}\left(\mathrm{NO}_{3}\right)_{2} \cdot 6 \mathrm{H}_{2} \mathrm{O}$ solution before adding 2-MeIm decreased the number of crystal nucleation sites, which led to large ZIF-8 single crystals. In contrast, the strong binding between CNTs and 2-MeIm group resulted in the formation of enough nucleation sites to lead to small ZIF-8 crystals in the composite materials. This study reports a very simple and efficient way to tune the number of nucleation sites for ZIF-8 crystals on CNTs, thereby allowing control over the ZIF-8 crystal size in the composite materials. The promising approach reported herein can pave the way for the design of metal-organic frameworks with specified size for targeted applications. 
Supplementary Materials: The following are available online at http:/ / www.mdpi.com/2073-4352/8/10/367/ s1, Figure S1: PXRD patterns of simulated ZIF-8 and synthesized ZIF-8/CNT composites under different reaction conditions, Figure S2: Major and minor phases in ZIF/CNT composite materials. PDF numbers for $\mathrm{Zn}_{3}(\mathrm{OH})_{4}\left(\mathrm{NO}_{3}\right)_{2}$ and $\mathrm{Zn}\left(\mathrm{NO}_{3}\right)_{2} \cdot 6 \mathrm{H}_{2} \mathrm{O}$ are 52-0627 and 19-1464, respectively, Figure S3: Top view of a single layer of graphene used in DFT calculations. Three possible adsorption positions are shown in this figure, Figure S4: SEM images of the ZIF-8/CNT composite prepared by adding (a) PVP, (b) SDS, and (c) CTAB, Figure S5: FT-IR spectra of ZIF-8/CNT synthesized with and without the addition of PVP, Figure S6: SEM image of the ZIF-8/CNT composite synthesized by adding 2-methylimidazole solution into $\mathrm{Zn}\left(\mathrm{NO}_{3}\right)_{2} \cdot 6 \mathrm{H}_{2} \mathrm{O}$ solution premixed with CNTs. The additive agent is PVP, Figure S7: SEM image (a) with size distribution histograms (b) for the ZIF-8/CNT composite prepared using 2-MeIm $/ \mathrm{Zn}^{2+}=70$ without any additive agent.

Author Contributions: B.Z. conceived the original idea; F.F. carried out the experiments and the simulations, analyzed the data, and wrote the paper; L.-H.X., H.D. and S.D. contributed the useful and deep discussions and modified the manuscript; Z.D. conducted the nitrogen sorption experiments.

Funding: This research was funded by the National Natural Science Foundation of China (NSFC) (Grants NO. 21503165 and 21601008), Shaanxi Province 100 plan, and the Science and Technology Foundation of Henan Province (Grants NO. 172102310043).

Conflicts of Interest: The authors declare no conflict of interest.

\section{References}

1. Park, K.S.; Ni, Z.; Côté, A.P.; Choi, J.Y.; Huang, R.; Uribe-Romo, F.J.; Chae, H.K.; O’Keeffe, M.; Yaghi, O.M. Exceptional chemical and thermal stability of zeolitic imidazolate frameworks. Proc. Natl. Acad. Sci. USA 2006, 103, 10186-10191. [CrossRef] [PubMed]

2. Li, J.-R.; Ma, Y.; McCarthy, M.C.; Sculley, J.; Yu, J.; Jeong, H.-K.; Balbuena, P.B.; Zhou, H.-C. Carbon dioxide capture-related gas adsorption and separation in metal-organic frameworks. Coord. Chem. Rev. 2011, 255, 1791-1823. [CrossRef]

3. Huang, X.C.; Lin, Y.Y.; Zhang, J.P.; Chen, X.M. Ligand-directed strategy for zeolite-type metal-organic frameworks: Zinc(II) imidazolates with unusual zeolitic topologies. Angew. Chem. Int. Ed. 2006, 45, 1557-1559. [CrossRef] [PubMed]

4. Treacy, M.M.J.; Ebbesen, T.W.; Gibson, J.M. Exceptionally high Young's modulus observed for individual carbon nanotubes. Nature 1996, 381, 678. [CrossRef]

5. Ebbesen, T.W.; Lezec, H.J.; Hiura, H.; Bennett, J.W.; Ghaemi, H.F.; Thio, T. Electrical conductivity of individual carbon nanotubes. Nature 1996, 382, 54. [CrossRef]

6. Wu, Z.; Chen, Z.; Du, X.; Logan, J.M.; Sippel, J.; Nikolou, M.; Kamaras, K.; Reynolds, J.R.; Tanner, D.B.; Hebard, A.F.; et al. Transparent, conductive carbon nanotube films. Science 2004, 305, 1273-1276. [CrossRef] [PubMed]

7. Zhang, K.; Lively, R.P.; Dose, M.E.; Brown, A.J.; Zhang, C.; Chung, J.; Nair, S.; Koros, W.J.; Chance, R.R. Alcohol and water adsorption in zeolitic imidazolate frameworks. Chem. Commun. 2013, 49, 3245-3247. [CrossRef] [PubMed]

8. Li, L.; Yao, J.; Xiao, P.; Shang, J.; Feng, Y.; Webley, P.A.; Wang, H. One-step fabrication of ZIF-8/Polymer composite spheres by a phase inversion method for gas adsorption. Colloid Polym. Sci. 2013, 291, 2711-2717. [CrossRef]

9. Zhang, C.; Lively, R.P.; Zhang, K.; Johnson, J.R.; Karvan, O.; Koros, W.J. Unexpected molecular sieving properties of zeolitic imidazolate framework-8. J. Phys. Chem. Lett. 2012, 3, 2130-2134. [CrossRef] [PubMed]

10. Liu, X.L.; Li, Y.S.; Zhu, G.Q.; Ban, Y.J.; Xu, L.Y.; Yang, W.S. An organophilic pervaporation membrane derived from metal-organic framework nanoparticles for efficient recovery of bio-alcohols. Angew. Chem. Int. Ed. 2011, 50, 10636-10639. [CrossRef] [PubMed]

11. Kim, D.; Kim, D.W.; Hong, W.G.; Coskun, A. Graphene/ZIF-8 composites with tunable hierarchical porosity and electrical conductivity. J. Mater. Chem. A 2016, 4, 7710-7717. [CrossRef]

12. Chen, B.; Zhu, Y.; Xia, Y. Controlled in situ synthesis of graphene oxide/zeolitic imidazolate framework composites with enhanced $\mathrm{CO}_{2}$ uptake capacity. RSC Adv. 2015, 5, 30464-30471. [CrossRef]

13. Kumar, R.; Jayaramulu, K.; Maji, T.K.; Rao, C.N.R. Hybrid nanocomposites of ZIF-8 with graphene oxide exhibiting tunable morphology, significant $\mathrm{CO}_{2}$ uptake and other novel properties. Chem. Commun. 2013, 49, 4947-4949. [CrossRef] [PubMed] 
14. Zhou, Y.; Zhou, L.; Zhang, X.; Chen, Y. Preparation of zeolitic imidazolate framework-8/graphene oxide composites with enhanced VOCs adsorption capacity. Microporous Mesoporous Mater. 2016, 225, 488-493. [CrossRef]

15. Zhang, W.; Tan, Y.; Gao, Y.; Wu, J.; Hu, J.; Stein, A.; Tang, B. Nanocomposites of zeolitic imidazolate frameworks on graphene oxide for pseudocapacitor applications. J. Appl. Electrochem. 2016, 46, 441-450. [CrossRef]

16. Zhou, H.; Zhang, J.; Zhang, J.; Yan, X.; Shen, X.; Yuan, A. High-capacity room-temperature hydrogen storage of zeolitic imidazolate framework/graphene oxide promoted by platinum metal catalyst. Int. J. Hydrog. Energy 2015, 40, 12275-12285. [CrossRef]

17. Petit, C.; Bandosz, T.J. Synthesis, characterization, and ammonia adsorption properties of mesoporous metal-organic framework (MIL(Fe))-graphite oxide composites: Exploring the limits of materials fabrication. Adv. Funct. Mater. 2011, 21, 2108-2117. [CrossRef]

18. Biswal, B.P.; Shinde, D.B.; Pillai, V.K.; Banerjee, R. Stabilization of graphene quantum dots (GQDs) by encapsulation inside zeolitic imidazolate framework nanocrystals for photoluminescence tuning. Nanoscale 2013, 5, 10556-10561. [CrossRef] [PubMed]

19. Dumée, L.; He, L.; Hill, M.; Zhu, B.; Duke, M.; Schutz, J.; She, F.; Wang, H.; Gray, S.; Hodgson, P.; Kong, L. Seeded growth of ZIF-8 on the surface of carbon nanotubes towards self-supporting gas separation membranes. J. Mater. Chem. A 2013, 1, 9208-9214. [CrossRef]

20. Yang, Y.; Ge, L.; Rudolph, V.; Zhu, Z. In Situ synthesis of zeolitic imidazolate frameworks/carbon nanotube composites with enhanced $\mathrm{CO}_{2}$ adsorption. Dalton Trans. 2014, 43, 7028-7036. [CrossRef] [PubMed]

21. Yoo, J.; Lee, S.; Lee, C.K.; Kim, C.; Fujigaya, T.; Park, H.J.; Nakashima, N.; Shim, J.K. Homogeneous decoration of zeolitic imidazolate framework-8 (ZIF-8) with core-shell structures on carbon nanotubes. RSC Adv. 2014, 4, 49614-49619. [CrossRef]

22. Abdi, J.; Vossoughi, M.; Mahmoodi, N.M.; Alemzadeh, I. Synthesis of metal-organic framework hybrid nanocomposites based on GO and CNT with high adsorption capacity for dye removal. Chem. Eng. J. 2017, 326, 1145-1158. [CrossRef]

23. Li, X.; Hao, C.; Tang, B.; Wang, Y.; Liu, M.; Wang, Y.; Zhu, Y.; Lu, C.; Tang, Z. Supercapacitor electrode materials with hierarchically structured pores from carbonization of MWCNTs and ZIF-8 composites. Nanoscale 2017, 9, 2178-2187. [CrossRef] [PubMed]

24. Zhang, Y.; Lin, B.; Wang, J.; Tian, J.; Sun, Y.; Zhang, X.; Yang, H. All-solid-state asymmetric supercapacitors based on $\mathrm{ZnO}$ quantum dots/carbon/CNT and porous N-doped carbon/CNT electrodes derived from a single ZIF-8/CNT template. J. Mater. Chem. A 2016, 4, 10282-10293. [CrossRef]

25. Sun, H.; Tang, B.; Wu, P. Two-dimensional zeolitic imidazolate fFramework/carbon nanotube hybrid networks modified proton exchange membranes for improving transport properties. ACS Appl. Mater. Interfaces 2017, 9, 35075-35085. [CrossRef] [PubMed]

26. Liu, Y.; Li, G.; Chen, Z.; Peng, X. CNT-threaded N-doped porous carbon film as binder-free electrode for high-capacity supercapacitor and Li-S battery. J. Mater. Chem. A 2017, 5, 9775-9784. [CrossRef]

27. Yue, Y.; Guo, B.; Qiao, Z.-A.; Fulvio, P.F.; Chen, J.; Binder, A.J.; Tian, C.; Dai, S. Multi-wall carbon nanotube@ zeolite imidazolate framework composite from a nanoscale zinc oxide precursor. Microporous Mesoporous Mater. 2014, 198, 139-143. [CrossRef]

28. Wang, Y.; Chen, B.; Zhang, Y.; Fu, L.; Zhu, Y.; Zhang, L.; Wu, Y. ZIF-8@MWCNT-derived carbon composite as electrode of high performance for supercapacitor. Electrochim. Acta 2016, 213, 260-269. [CrossRef]

29. Wan, L.; Shamsaei, E.; Easton, C.D.; Yu, D.; Liang, Y.; Chen, X.; Abbasi, Z.; Akbari, A.; Zhang, X.; Wang, H. ZIF-8 derived nitrogen-doped porous carbon/carbon nanotube composite for high-performance supercapacitor. Carbon 2017, 121, 330-336. [CrossRef]

30. Zhou, J.; Yu, X.; Fan, X.; Wang, X.; Li, H.; Zhang, Y.; Li, W.; Zheng, J.; Wang, B.; Li, X. The impact of the particle size of a metal-organic framework for sulfur storage in Li-S batteries. J. Mater. Chem. A 2015, 3, 8272-8275. [CrossRef]

31. Zaręba, J.K.; Nyk, M.; Samoć, M. Co/ZIF-8 heterometallic nanoparticles: control of nanocrystal size and properties by a mixed-metal approach. Cryst. Growth Des. 2016, 16, 6419-6425. [CrossRef]

32. Enomoto, T.; Ueno, S.; Hosono, E.; Hagiwara, M.; Fujihara, S. Size-controlled synthesis of ZIF-8 particles and their pyrolytic conversion into $\mathrm{ZnO}$ aggregates as photoanode materials of dye-sensitized solar cells. CrystEngComm 2017, 19, 2844-2851. [CrossRef] 
33. Zhu, Y.; Ciston, J.; Zheng, B.; Miao, X.; Czarnik, C.; Pan, Y.; Sougrat, R.; Lai, Z.; Hsiung, C.-E.; Yao, K.; et al. Unravelling surface and interfacial structures of a metal-organic framework by transmission electron microscopy. Nat. Mater. 2017, 16, 532-536. [CrossRef] [PubMed]

34. Perdew, J.P.; Burke, K.; Ernzerhof, M. Generalized gradient approximation made simple. Phys. Rev. Lett. 1996, 77, 3865-3868. [CrossRef] [PubMed]

35. Blöchl, P.E. Projector augmented-wave method. Phys. Rev. B 1994, 50, 17953-17979. [CrossRef]

36. Kresse, G.; Joubert, D. From ultrasoft pseudopotentials to the projector augmented-wave method. Phys. Rev. B 1999, 59, 1758-1775. [CrossRef]

37. Kresse, G.; Furthmüller, J. Efficient iterative schemes for ab initio total-energy calculations using a plane-wave basis set. Phys. Rev. B 1996, 54, 11169-11186. [CrossRef]

38. Kresse, G.; Hafner, J. Ab initio molecular-dynamics simulation of the liquid-metal-amorphous-semiconductor transition in germanium. Phys. Rev. B 1994, 49, 14251-14269. [CrossRef]

39. Huang, M.-Z.; Ching, W.Y. Calculation of optical excitations in cubic semiconductors. I. Electronic structure and linear response. Phys. Rev. B 1993, 47, 9449-9463. [CrossRef]

40. Kresse, G.; Furthmüller, J. Efficiency of ab-initio total energy calculations for metals and semiconductors using a plane-wave basis set. Comput. Mater. Sci. 1996, 6, 15-50. [CrossRef]

41. Pan, Y.; Liu, Y.; Zeng, G.; Zhao, L.; Lai, Z. Rapid synthesis of zeolitic imidazolate framework-8 (ZIF-8) nanocrystals in an aqueous system. Chem. Commun. 2011, 47, 2071-2073. [CrossRef] [PubMed]

42. Gross, A.F.; Sherman, E.; Vajo, J.J. Aqueous room temperature synthesis of cobalt and zinc sodalite zeolitic imidizolate frameworks. Dalton Trans. 2012, 41, 5458-5460. [CrossRef] [PubMed]

43. Kida, K.; Okita, M.; Fujita, K.; Tanaka, S.; Miyake, Y. Formation of high crystalline ZIF-8 in an aqueous solution. CrystEngComm 2013, 15, 1794-1801. [CrossRef]

44. Cravillon, J.; Münzer, S.; Lohmeier, S.-J.; Feldhoff, A.; Huber, K.; Wiebcke, M. Rapid room-temperature synthesis and characterization of nanocrystals of a prototypical zeolitic imidazolate framework. Chem. Mater. 2009, 21, 1410-1412. [CrossRef]

45. Wang, J.; Yang, C.; Huang, Z.; Humphrey, M.G.; Jia, D.; You, T.; Chen, K.; Yang, Q.; Zhang, C. Seed-catalyzed heteroepitaxial growth and nonlinear optical properties of zinc selenide nanowires. J. Mater. Chem. 2012, 22, 10009-10014. [CrossRef]

46. Lu, G.; Li, S.; Guo, Z.; Farha, O.K.; Hauser, B.G.; Qi, X.; Wang, Y.; Wang, X.; Han, S.; Liu, X.; et al. Imparting functionality to a metal-organic framework material by controlled nanoparticle encapsulation. Nat. Chem. 2012, 4, 310. [CrossRef] [PubMed]

47. O'Connell, M.J.; Boul, P.; Ericson, L.M.; Huffman, C.; Wang, Y.; Haroz, E.; Kuper, C.; Tour, J.; Ausman, K.D.; Smalley, R.E. Reversible water-solubilization of single-walled carbon nanotubes by polymer wrapping. Chem. Phys. Lett. 2001, 342, 265-271. [CrossRef]

48. He, C.-T.; Jiang, L.; Ye, Z.-M.; Krishna, R.; Zhong, Z.-S.; Liao, P.-Q.; Xu, J.; Ouyang, G.; Zhang, J.-P.; Chen, X.-M. Exceptional hydrophobicity of a large-pore metal-organic zeolite. J. Am. Chem. Soc. 2015, 137, 7217-7223. [CrossRef] [PubMed]

49. Li, F.; Wang, Y.; Wang, D.; Wei, F. Characterization of single-wall carbon nanotubes by $\mathrm{N}_{2}$ adsorption. Carbon 2004, 42, 2375-2383. [CrossRef]

(C) 2018 by the authors. Licensee MDPI, Basel, Switzerland. This article is an open access article distributed under the terms and conditions of the Creative Commons Attribution (CC BY) license (http://creativecommons.org/licenses/by/4.0/). 